\title{
Pulsars, SKA and Time-Domain Studies in the Future
}

\author{
Michael Kramer ${ }^{1,2}$ \\ ${ }^{1}$ Max-Planck-Institut für Radioastronomie, 53121 Bonn, Germany \\ ${ }^{2}$ Jodrell Bank Centre for Astrophysics, University of Manchester, Manchester, M13 9PL, UK \\ email: mkramer@mpifr.de \\ Invited Talk
}

\begin{abstract}
Pulsars are a classical example for time-domain astronomy. Using just the precisely measured arrival time of pulses from pulsars, we can study a wide range of physics, and in particular probe the validity of theories of gravity. Despite huge success, pulsar astrophysics will completely change with the Square Kilometre Array (SKA), lifting pulsar science into a new era of time-domain astronomy. I will review the applications using pulsars and the prospects for the SKA.
\end{abstract}

Keywords. (stars:) pulsars: general, gravitation, gravitational waves

\section{Introduction}

The Square Kilometre Array (SKA) will be the world's largest radio telescope, enabling transformational science. It will be a flexible, multi-purpose observatory that will be able to serve the whole astronomy community as the premier imaging and surveying instrument. Five Key Science Projects have been selected by the international community; those projects must drive essential parts of the design in order to ensure that their science goals can be achieved. Some of the most major advances in science can be expected for time-domain studies and pulsars in particular. The reason is the SKA's overwhelming sensitivity by virtue of its enormous collecting area, and is its defining feature. As the name suggests, the total collecting area for the SKA is planned to be close to one square kilometre, offering a target sensitivity of $2 \times 10^{4} \mathrm{~m}^{2} \mathrm{~K}^{-1}$.

\section{The Square Kilometre Array}

The SKA is likely to consist of sparse Aperture Arrays (AAs) of tiled dipoles in the frequency range of 70 to $500 \mathrm{MHz}$, while above $500 \mathrm{MHz}$ the following three implementations are being considered:

a) $300015-\mathrm{m}$ dishes with a single pixel feed, a sensitivity of $0.6 \mathrm{SKA}$ units, $T_{\mathrm{sys}}=$ $30 \mathrm{~K}$ and $70 \%$ efficiency covering the frequency range of $500 \mathrm{MHz}$ to $10 \mathrm{GHz}$.

b) $200015-\mathrm{m}$ dishes with Focal Plane Arrays (FPAs) from $500 \mathrm{MHz}$ to $1.5 \mathrm{GHz}$, a sensitivity of 0.35 SKA units, a Field-of-View (FoV) of $20 \mathrm{deg}^{2}, T_{\text {sys }}=35 \mathrm{~K}$ and $70 \%$ efficiency and a single pixel feed from 1.5 to $10 \mathrm{GHz}$, with $T_{\mathrm{sys}}=30 \mathrm{~K}$.

c) A combination of dense AAs with a FoV of $250 \mathrm{deg}^{2}$, a sensitivity of $0.5 \mathrm{SKA}$ units, covering the frequency range of 500 to $800 \mathrm{MHz}$ and $240015-\mathrm{m}$ dishes with a single pixel feed covering the frequency range of $800 \mathrm{MHz}$ to $10 \mathrm{GHz}$, a sensitivity of 0.5 SKA units, $T_{\text {sys }}=30 \mathrm{~K}$ and $70 \%$ efficiency. 
The signals of these receiving elements can be combined to form independent FoVs, resulting in a survey speed of 10,000 times that of existing telescopes.

The exact configuration of the SKA will be determined after the present global R \& D phase is completed. However, most of the collecting area will be concentrated in a central core of the SKA: $20 \%$ of the collecting area will be located within a 1-km radius, and $50 \%$ within a $5-\mathrm{km}$ radius. The remainder of the receiving elements will be distributed in stations (probably) along log-spiral arms with base-lines extending to 3,000 $\mathrm{km}$ in order to permit high-resolution imaging and high-precision astrometry too. Increased resolution will be achieved via intercontinental base-lines between Australia and Southern Africa.

The SKA will be built in three phases. Phase I: by 2010, about 0.1 SKA unit will be available in the core region covering the low- and mid-frequencies. Phase II: by 2022, the full array will be completed. Phase III: Beyond 2022, high-frequency capabilities for observations, potentially up to $\sim 20 \mathrm{GHz}$, will be added.

\section{Time-Domain Radio Astronomy}

As demonstrated by other contributions in these proceedings (for instance, Keane, p. 342), the current phase space in time-domain astronomy is covered only sparsely by known sources. In the "coherent-emission" part of phase space, most of the sources represent the emission from neutron stars, and especially from pulsars. Indeed, the radio emission from pulsars shows a rich variety of intriguing properties with a wide range of observed time-scales. The observed phenomena, which cover an astounding 18 orders of magnitude of time, have been summarised by Stappers (p. 103). The range of physics that has been probed is similarly wide, extending from gravitational physics to extreme plasma physics, from solid state physics to studies of the Galactic structure, from stellar physics to binary evolution. The SKA will contribute enormously to all these areas. Its contribution to studies of gravitational physics by measuring pulsars orbiting black holes or by using them as cosmic gravitational-wave detectors has been identified as one of five SKA Key Science Projects (see Kramer et al. 2004). The questions to be addressed and answered include:

- What is the nature of gravity? Was Einstein right? Is gravity described by a tensor field or are there additional scalar fields, as it is sometimes proposed to explain Dark Energy?

- What are the properties of gravitational waves? Do gravitons have Spin 2? What is the mass of gravitons, and hence what is the propagation speed of gravitational waves?

- What happens in strong gravitational fields, in conditions of extreme curvature and near singularities? What are the properties of black holes? Do the "no-hair" and "cosmic censorships" theorems hold?

Answering these questions requires a survey for pulsars and the high-precision timings of a selected sample of them.

\section{A Galactic Census for Pulsars}

We currently know about 2,000 pulsars; they include 10 double neutron stars and one double pulsar, but no pulsar-black hole systems. Major continuing efforts to increase the numbers include the P-ALFA survey with the Arecibo telescope, the GBT-350 $\mathrm{MHz}$ survey or the High Time Resolution Universe (HTRU) Survey in the Northern hemisphere with the Effelsberg 100-m telescope, and in the Southern hemisphere with the Parkes telescope. Recently, deep searches of unidentified point sources seen by the 


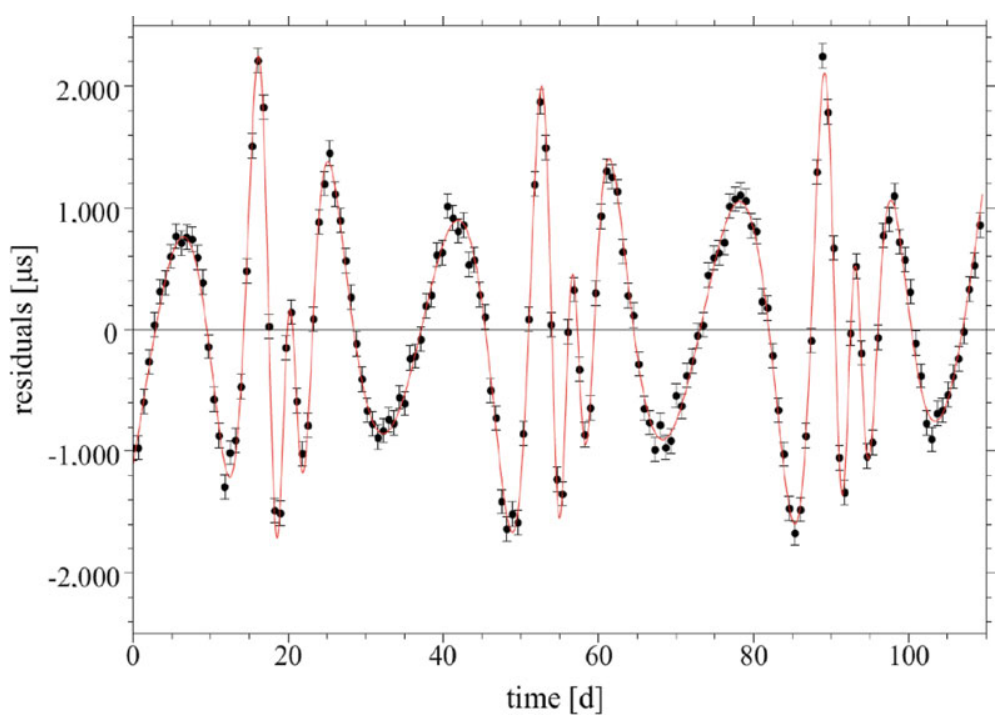

Figure 1. Timing residuals of a pulsar orbiting the super-massive BH in the Galactic Centre in a $0.1-y r$ orbit. Shown are three orbits with an eccentricity of $e=0.4$ for an extreme Kerr BH. Even with a timing precision of only $100 \mu \mathrm{s}$, the characteristic periodic residuals caused by the BH's quadrupole moment are clearly visible (Liu et al. 2012).

FERMI gamma-ray space telescope have been tremendously successful. Soon, the Low Frequency Array (LOFAR) should identify many new pulsars in the solar vicinity (van Leeuwen \& Stappers 2010), providing a local census. Still, all these very good results are only a prelude to what will be possible with the SKA.

Depending on the low end of the luminosity function and the collecting area that is deployable, one can find practically all active Galactic pulsars beaming towards Earth, providing a "Galactic Census" of pulsars (Cordes et al. 2004). We expect to find about 20,000 to 30,000 pulsars, including 1,000 to 3,000 millisecond pulsars and 100 relativistic binaries (Smits et al. 2009). This survey will be conducted at a frequency between 1-3 GHz, depending on Galactic latitude and frequency overlap of different antenna types. The outcome of the survey will be a sample of accurate millisecond pulsar clocks that we will time as a Pulsar Timing Array to study the nHz-gravitational wave sky. Furthermore, we should uncover the rare pulsar-black hole $(\mathrm{BH})$ systems where a pulsar orbits a stellar $\mathrm{BH}$ in the Galactic plane or an intermediate $\mathrm{BH}$ in a globular cluster. Once the interesting sources have been identified, they will need to be timed with the full array to achieve the best possible precision. The timing therefore consists of two parts: a medium to low-precision timing programme to follow-up the 20,000 to 30,000 discovered sources to identify the "goodies", and a high-precision timing part of an estimated 100 to 300 sources in order to extract the science. For the latter sources, we can expect timing precisions to increase with the sensitivity of the SKA over the performance of current telescopes.

While the Galactic Census will deliver the bulk of the sources, a small piece of skythe Galactic Centre (GC) - needs to be treated specially. The GC region is interesting not only because a large stellar density suggests the occurrence of BH-MSP systems, but also because we will discover pulsars orbiting the super-massive BH in the GC. Tracing the movement and rotation of pulsars in their orbits provides in principle an easier measurement of the $\mathrm{BH}$ spin and quadrupole moment since those quantities scale with the square and cube of the masses, respectively. Such a measurement would also extend 


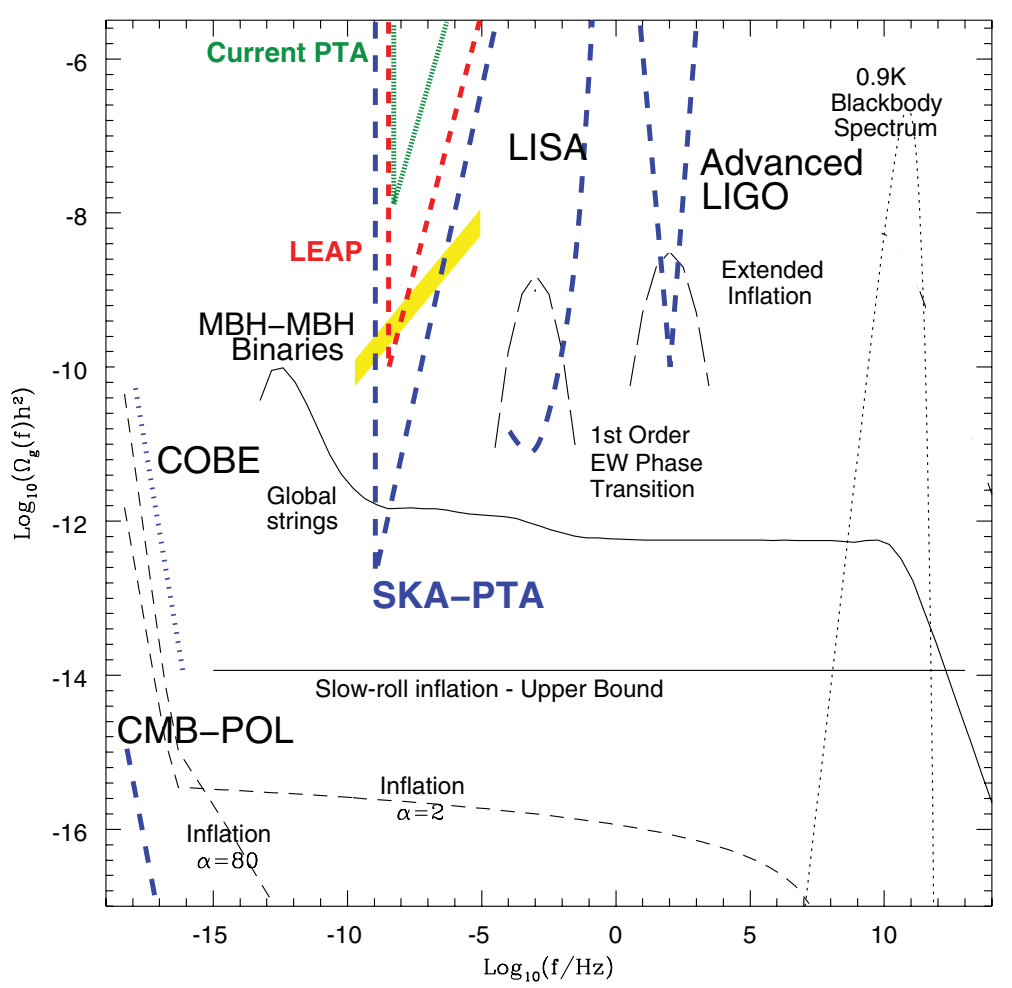

Figure 2. Summary of the potential cosmological sources of a stochastic gravitational-wave (GW) background, overlaid with bounds from COBE, current Pulsar Timing Array (PTA) experiments and the goals of CMB polarization experiments, LISA and Advanced LIGO. LEAP will improve the current best PTA limits by more than two orders of magnitude, enabling the detection of a GW background caused by the merger of massive black holes (MBHs) in early galaxy formation. The amplitude depends on the MBH mass function and merger rate, so uncertainty is indicated by the size of the shaded/yellow area. LEAP is the next logical step towards realizing a PTA with the SKA; the latter will improve the current sensitivity by about four orders of magnitude.

the mass range of $\mathrm{BH}$ properties studied, but the problem is that the interstellar medium in the GC causes severe interstellar scattering because of multi-path propagation. That can only be combated by observing at higher radio frequencies; frequencies between 10 and $15 \mathrm{GHz}$ should be sufficient.

\section{Black-Hole Properties}

What makes a binary pulsar with a black-hole companion so interesting is that it has the potential to provide a superb new probe of relativistic gravity. As pointed out by Damour \& Esposito-Farèse (1998), the discriminating power of this probe might supersede all its present and foreseeable competitors. The reason lies in the fact that such a system would be very sensitive to strong gravitational self-field effects, making it (for instance) an excellent probe for tensor-scalar theories. Wex \& Kopeikin (1999) showed that, in principle, the measurement of classical and relativistic spin-orbit coupling in a pulsar$\mathrm{BH}$ binary allows us to determine the spin and the quadrupole moment of the black hole. That would test the "cosmic censorship" conjecture and the "no-hair" theorem. While Wex \& Kopeikin (1999) showed that, with current telescopes, such an experiment would be almost impossible to perform (with the possible exception of pulsars about the 


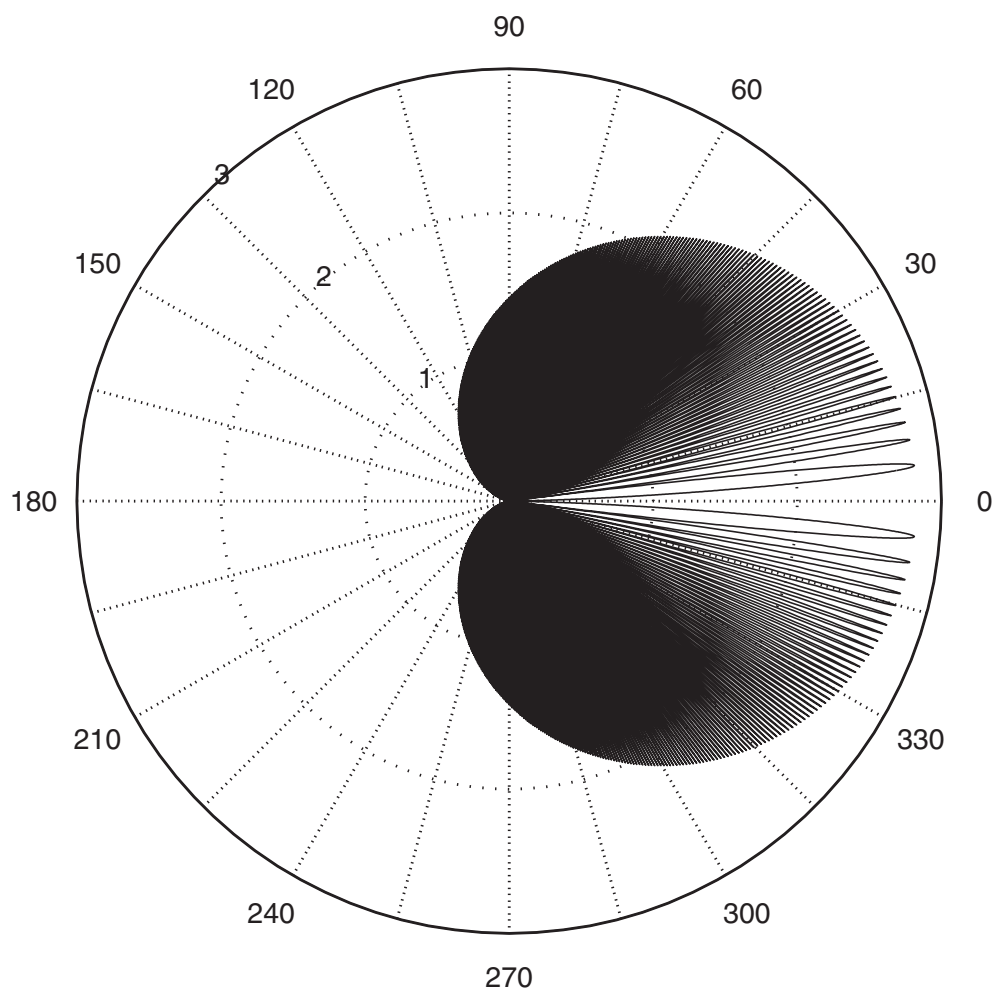

Figure 3. "Antenna pattern" of a single-pulsar timing response to a single monochromatic gravitational-wave source. For illustration purposes, the pulsar distance is chosen to be small with a value of $200 \mathrm{pc}$ and the GW period is chosen as 5 years, in order to show the structure of the response pattern. The GW source is in the $0^{\circ}$ angle position, the orbital plane inclination is $90^{\circ}$ and the orbital plane coincides with the plane of the paper (Lee et al. 2011). The fine structure of this antenna pattern enables the precise localisation of gravitational-wave sources.

Galactic-Centre black hole), Kramer et al. (2004) pointed out that the SKA sensitivity should be sufficient. Indeed, this experiment benefits from the SKA sensitivity in multiple ways. On the one hand it provides the required timing precision, but on the other it also allows us to perform the Galactic Census which should eventually deliver a sample of pulsars with BH companions. As shown recently by Liu et al. (2012), it should be "fairly easy" to measure the spin of the GC black hole with a precision of $10^{-4}-10^{-3}$. Even for a pulsar with a timing precision of only $100 \mu \mathrm{s}$, characteristic periodic residuals would enable the measurement of the $\mathrm{BH}$ quadrupole moment to $1 \%$ or better. That would allow us to test the no-hair theorem with similar precision (see Fig. 1). Perhaps most importantly, by using the method described by Liu et al. it will be possible to determine whether the pulsar-SGR A* system is "clean", i.e. if the timing data are undisturbed by a nearby stellar population.

\section{Gravitational-wave Astronomy}

While pulsars already provide indirect evidence for the existence of gravitational waves, they can also be used to detect and study them directly using a "Pulsar Timing Array" (PTA). Such PTA experiments have a good chance of detecting GWs, but we are currently limited by the sensitivity of our telescopes. The Large European Array for Pulsars (LEAP), which combines the collecting power of Europe's largest telescopes (Kramer 
\& Stappers 2010), may get very close to detecting, or may even detect, a stochastic gravitational-wave background, possibly with the help of the International Pulsar Timing Array (IPTA) (see Hobbs et al. 2010), but in order to guarantee a detection - or even study the properties of gravitational waves - we need the overwhelming sensitivity of the SKA. Owing to the increased timing precision achievable with the SKA and the large number of suitable sources to be discovered, a PTA with the SKA would achieve a sensitivity that even exceeds that of LISA (Kramer \& Stapppers 2010; see Fig. 2).

Different types of signals can be detected and studied with the SKA: stochastic, burst, and periodic signals. A stochastic gravitational-wave background should arise from a variety of sources. Cosmological sources include inflation, string cosmology, cosmic strings and phase transitions (Kramer et al. 2004). The expected correlation signal among the PTA pulsars can be measured with very high precision, so the polarisation and mass properties of gravitons can be probed and compared with the predictions of general relativity (Lee et al. 2008, 2010). For single GW sources, the superb astrometric precision that will be realised with the SKA (Smits et al. 2011) will enable us to pinpoint their locations to a precision that allows efficient electromagnetic follow-up (Lee et al. 2011; also see Fig. 3).

\section{Conclusions}

We have only touched upon a small amount of science topics that can and will be addressed by the SKA. Further topics range from studies of pulsar radio emission to attempts to understand the conditions for the formation of planets around pulsars, facilitated for the first time by having a Galactic Census of pulsars and hence a (nearly) complete sample. But already the SKA pathfinders will provide a great amount of exciting new discoveries, giving us a glimpse of what will be possible with the SKA. The global efforts will culminate in the construction of the SKA as the world's largest and most powerful telescope. As a versatile observatory it will revolutionise many areas of astrophysics, but in particular that of pulsars and fundamental physics.

\section{References}

Cordes, J. M., Kramer, M., Lazio, T. J. W., Stappers, B. W., Backer, D. C., \& Johnston, S., 2004, New Astr., 48, 1413

Damour, T. \& Esposito-Farèse, G., 1998, Phys. Rev. D, 58 (042001), 1

Hobbs, G., et al. 2010, Classical and Quantum Gravity, 27(8), 084013

Kramer, M. \& Stappers, B., 2010, in Proceedings of the ISKAF2010 Science Meeting, POS, 112, 34

Kramer, M., Backer, D. C., Cordes, J. M., Lazio, T. J. W.., Stappers, B. W., \& Johnston, S., $2004,48,993$

Lee, K. J., et al., 2011, MNRAS, 414, 3251

Lee, K. J., Jenet, F. A., \& Price, R. H., 2008, ApJ, 685, 1304

Liu, K., Wex, N., Kramer, M., Cordes, J. M., \& Lazio, T. J. W.. 2012, ApJ, in press

Smits, R., Kramer, M., Stappers, B., Lorimer, D. R., Cordes, J. M., \& Faulkner, A., 2009, A\&A, 493, 1161

Smits, R., Tingay, T., Wex, N., Kramer, M., \& Stappers, B. 2011, A\&\&A, 528, 108

van Leeuwen, J. \& Stappers, B. W., 2010, A\&\&A, 509, A7

Wex, N. \& Kopeikin, S., 1999, ApJ, 513, 388 